\title{
THE EFFECT OF DIFFERENT LEVELS OF POTASSIUM AND PHOSPHATE FERTILIZER ON GROWTH AND NITROGEN ,PHOSPHORUS AND POTASSIUM CONTENTS OF BEAN PLANT (Vicia faba $L$ )
}

ALmagrebi, N. M. H. ${ }^{1}$

Soil and Water Dept. ,Fac. of Agric. ,Sana Univ. , Yemen

تأثير مستويات مختلفة من السماد البوتاسي والفوسفاتي علي نمو ومحتوى نباتات

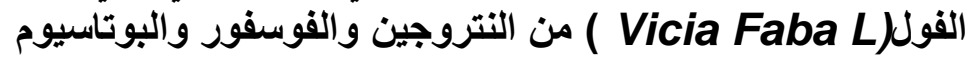
نجيب محمد حسين المغربي قسم الاراضي والمياة ـ كلية الزرراعة ـ جامعة صنعاءـ اليمن الملخص

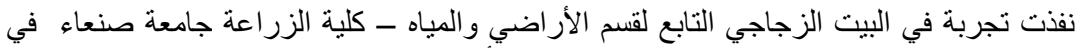

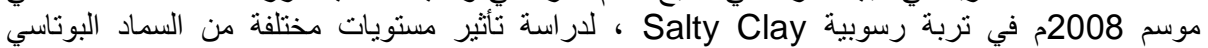

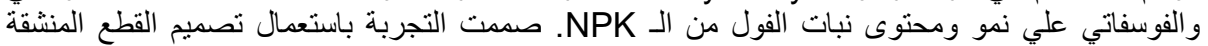
Split Plot Design

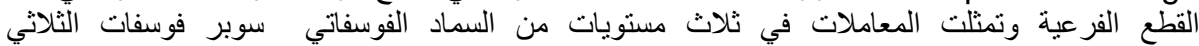

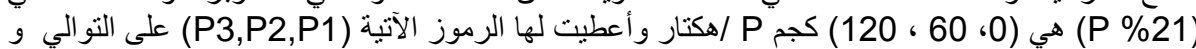

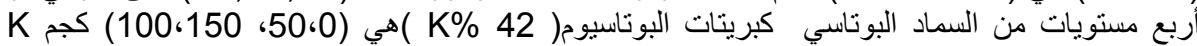

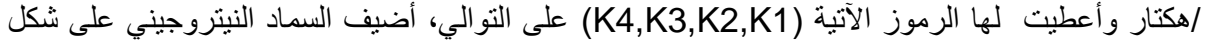

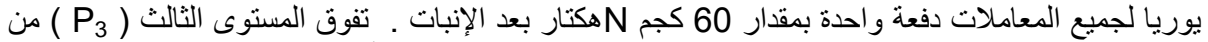

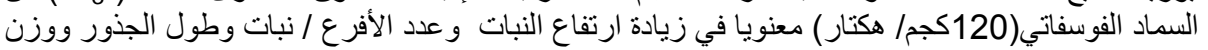

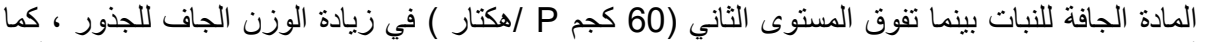

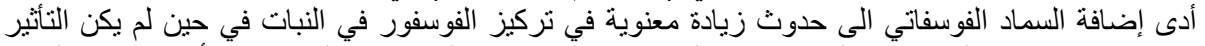

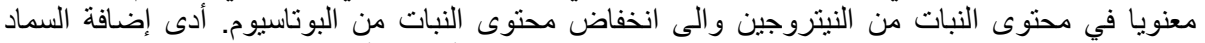

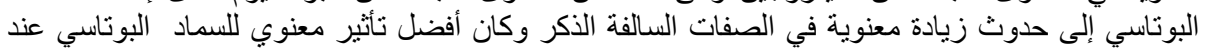

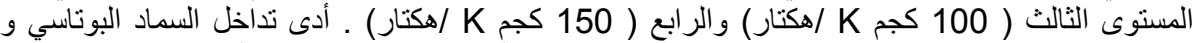

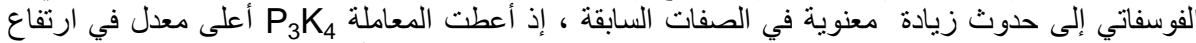

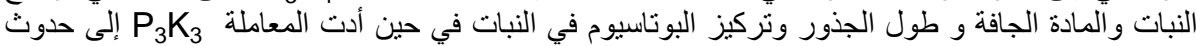

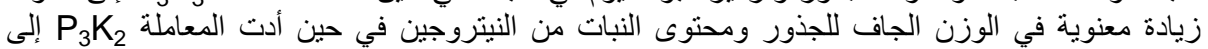

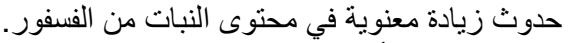

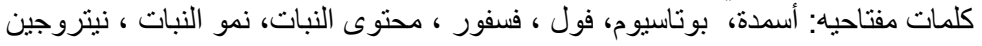

المقدمة

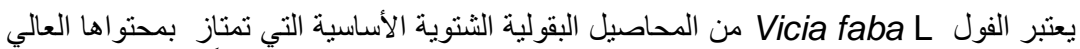

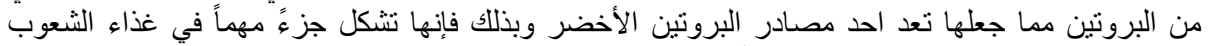

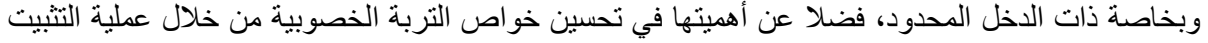

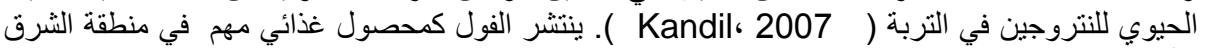

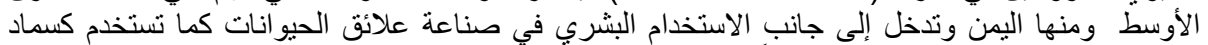

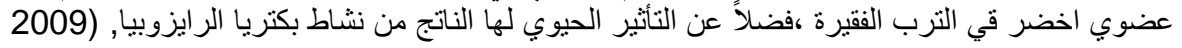

Associate Professor, Department of Soil and water, Faculty of Agriculture, University of ${ }^{1}$ Sana'a, Yemen 


\section{ALmagrebi, N. M. H.}

(Bensoltan وChafi

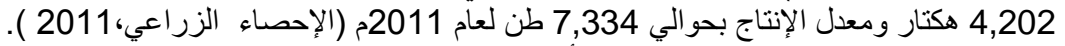

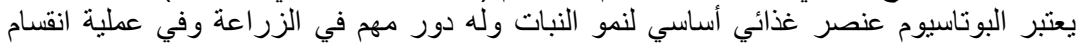

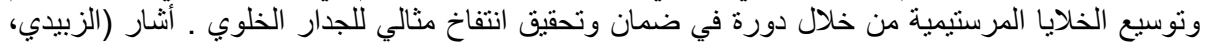

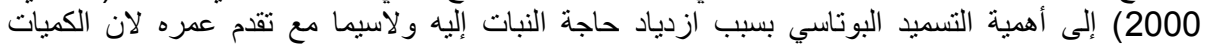

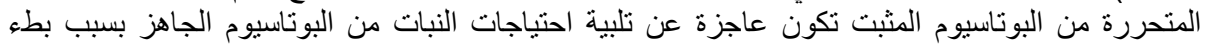

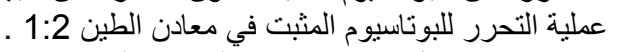

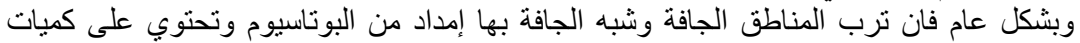

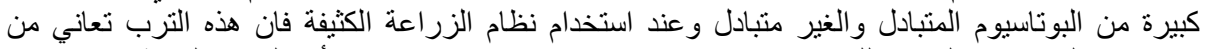

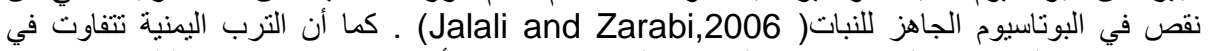

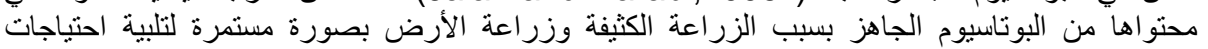

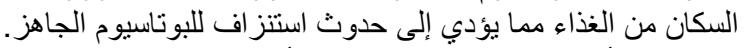

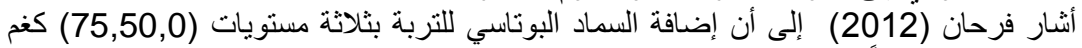

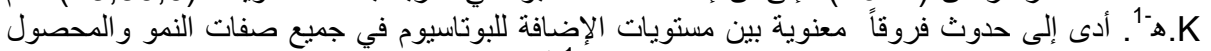

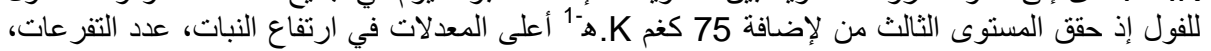

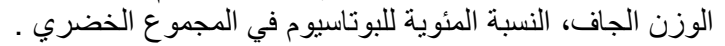

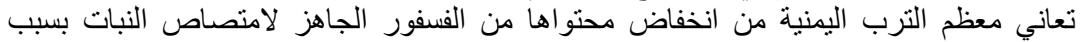

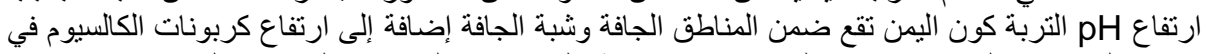

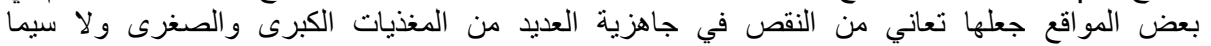

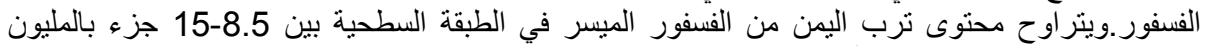

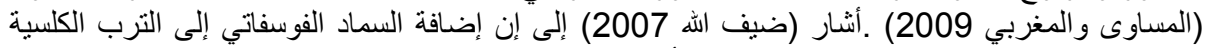

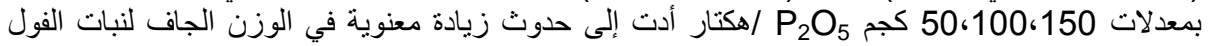

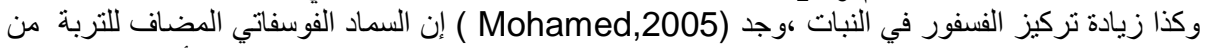

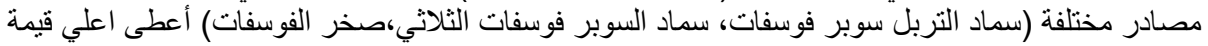

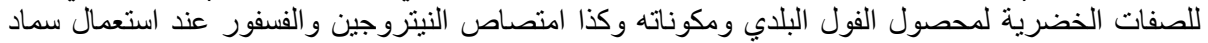

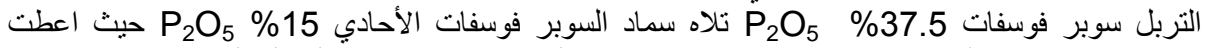

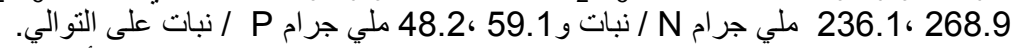

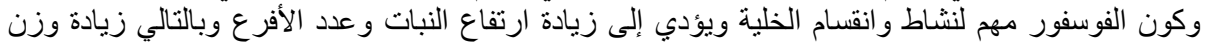

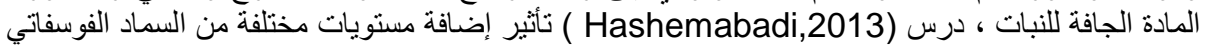

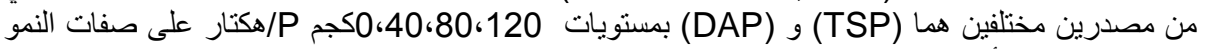

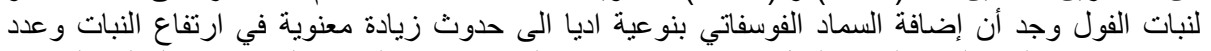

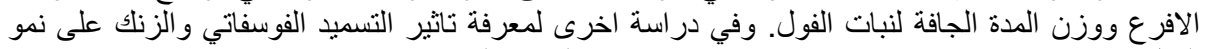

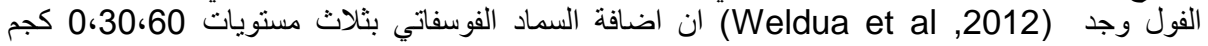

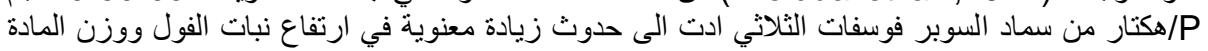

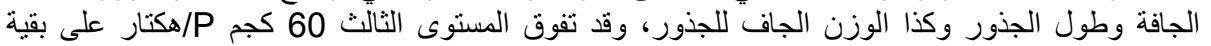

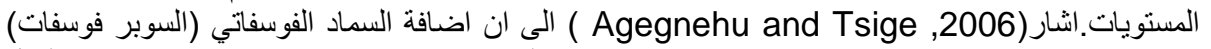

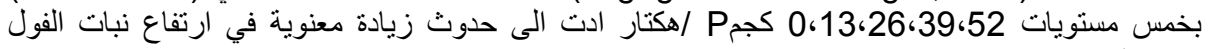

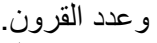
لذا بهوف البحث إلى دراسة تأثير مستويات مختلفة من السماد البوتاسي والفوسفاتي على نمو ومحتوى نبات الفول من النتروجين و الفوسفور و البوتاسيوم ـ 


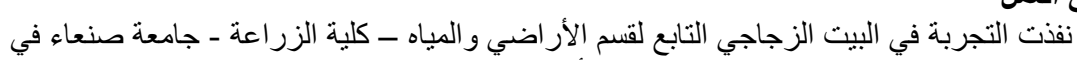

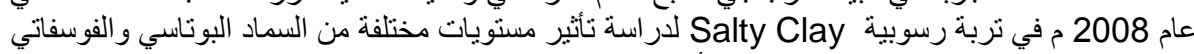

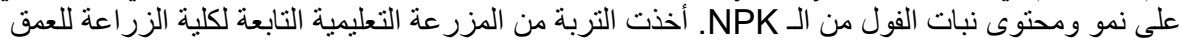
من 0 إلى 30 سم(جدول1يوضح بعض الخول الـ الكيمائية للترية المستخدمة في التجربة).

\begin{tabular}{|c|c|c|c|c|c|c|c|c|c|c|c|c|c|c|c|}
\hline \multirow{3}{*}{\begin{tabular}{|c|c|}
$\mathbf{K}$ \\
\end{tabular}} & & & & & הرנ & & & & & ثزب بـ & & (I) & حوا & $\Delta x$ & لدول \\
\hline & \multicolumn{2}{|c|}{ العناصر الجاهزة } & \multicolumn{6}{|c|}{ الكاتيونات والانيونات الذائبة } & \multicolumn{2}{|r|}{\begin{tabular}{|c|} 
Total \\
N
\end{tabular}} & O.M & \multirow[t]{2}{*}{$\mathrm{CacO}_{3}$} & \multirow[t]{2}{*}{ pH } & \multirow[t]{2}{*}{ EC } & \multirow[t]{2}{*}{ |الصفة | } \\
\hline & K & $\bar{P}$ & $\mathrm{~K}$ & SO4 & CL & $\mathrm{HCO}_{3}$ & $\mathrm{Na}$ & $M g$ & Ca & & & & & & \\
\hline $\mathrm{Cmc}$ & 1. $\mathrm{Kg}^{-1}$ & $\mathrm{mg} \cdot \mathrm{Kg}^{-1}$ & \multicolumn{6}{|c|}{$\mathrm{Kg}^{-1}$} & & $\%$ & $\%$ & $\%$ & & dS. $m^{-1}$ & الوحدة | \\
\hline 40 & 1.414 & 9 & \begin{tabular}{|l|}
0.014 \\
\end{tabular} & 06. & 0.28 & 0.31 & \begin{tabular}{|l|}
0.24 \\
\end{tabular} & 0.14 & 0.26 & 0.05 & 98. & 6.4 & 7.86 & 0.31 & ققيمة \\
\hline
\end{tabular}

ثم نخلت بمنذل قطر فتحاته 4مم و أضيفت إلى أصص بلاستكية سعه 7 كجم بواقع 5 كجم تربة /

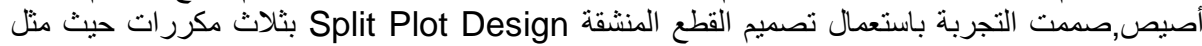

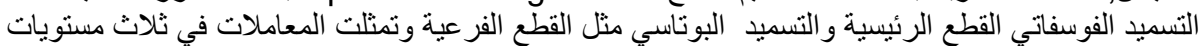

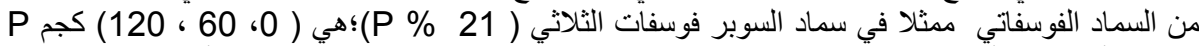

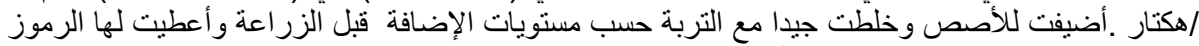

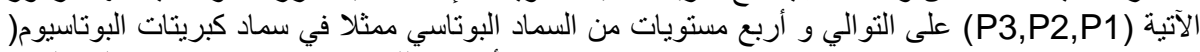

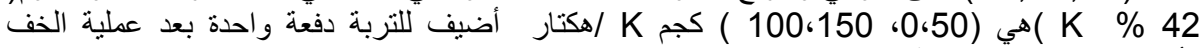

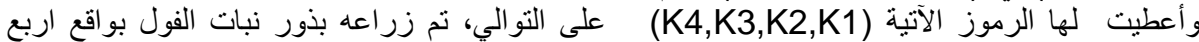

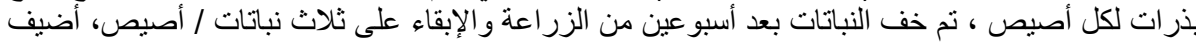

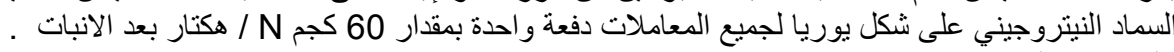

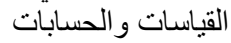
تم اخذ القياسات الآتية: ارتفاع النبات سم ،عدد الأفرع / نبات، الوزن الجاف للنباتات جرام /

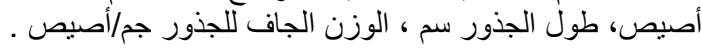

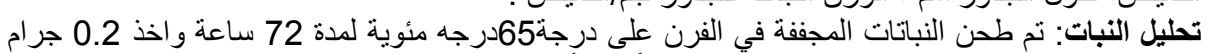

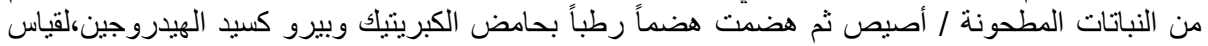

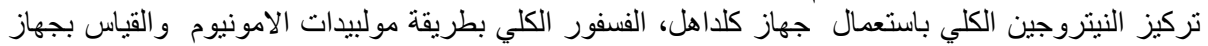
Spectrophotometer الواردة في (Page et al, 1982)

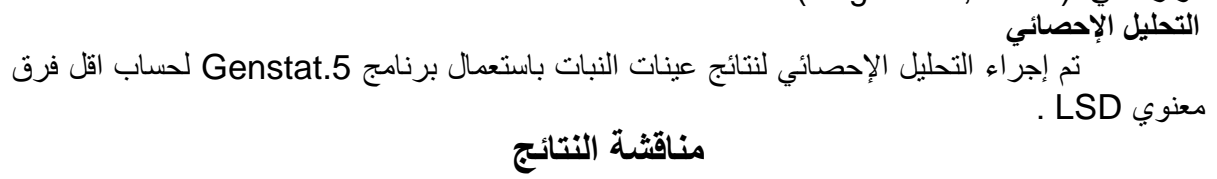
تشير نتائج التحليل الإحصائي جدول 2 إلى أن إضافة السماد الفوسفاتي أدى إلى حدوث زيادة معنوية

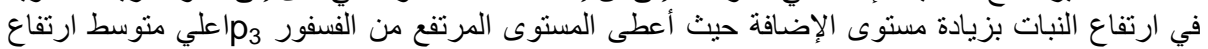

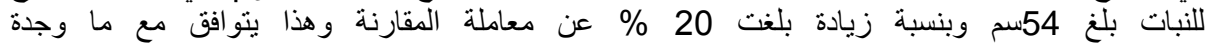

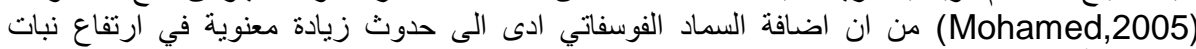

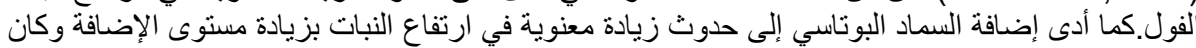

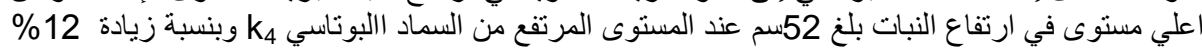

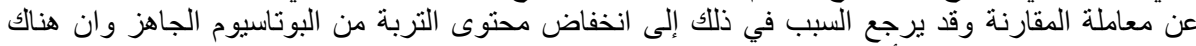

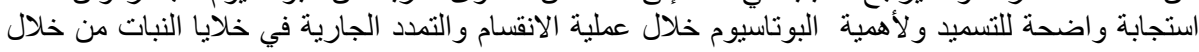

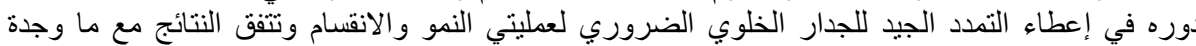

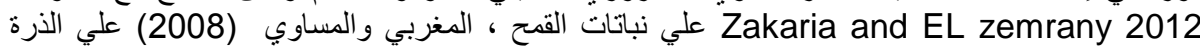
الثنامية و فرحان(2012) علي الفول. - مل 
جدول 2: تأثير إضافة السماد الفوسفاتي والبوتاسي وتداخلاتهما على ارتفاع النبات (سم)

\begin{tabular}{|c|c|c|c|c|}
\hline MEAN K & P3 & P2 & P1 & Treatment \\
\hline 46.6 & 51.5 & 47.5 & 40.9 & $\mathrm{~K} 1$ \\
\hline 48.9 & 53.1 & 48.9 & 44.8 & $\mathrm{~K} 2$ \\
\hline 49.5 & 54.9 & 49.0 & 44.7 & K3 \\
\hline 52.3 & 56.6 & 51.0 & 49.4 & K4 \\
\hline 5.35 & \multicolumn{3}{|c|}{10.21} & LSD \\
\hline & 54.0 & 49.1 & 44.95 & MEAN P \\
\hline & \multicolumn{3}{|c|}{5.53} & LSD \\
\hline
\end{tabular}

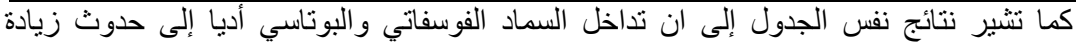

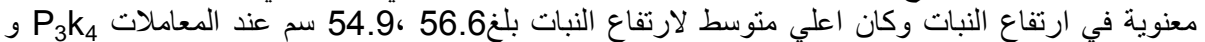

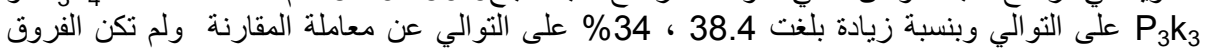

تشير نتائج تحليل الجدول3 إلى حدوث زيادة معنوية في عدد الأفرع للنباتات بزيادة مستوى إضافة معنوية فيما بينها.

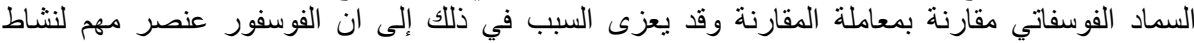

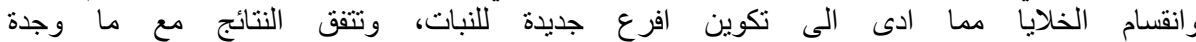

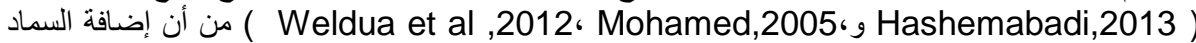
الفوسفاتي إلى التربة بمستويات مختلفة أدى إلى حدوث زيادة معنوية في عدد الأفرع لنبات الفول.

جدول 3 : تأثير إضافة السماد الفوسفاتي والبوتاسي وتداخلاتهما على متوسط عدد الأفرع للنباتات الفول.

\begin{tabular}{|c|c|c|r|r|}
\hline Mean K & P3 & P2 & P1 & Treatment \\
\hline 2.57 & 2.6 & 2.6 & 2.5 & K1 \\
\hline 2.67 & 3 & 2.4 & 2.6 & K2 \\
\hline 2.77 & 2.8 & 3.2 & 2.3 & K3 \\
\hline 2.63 & 2.7 & 2.6 & 2.7 & K4 \\
\hline 0.13 & \multicolumn{4}{|c|}{0.17} \\
\hline
\end{tabular}

كما إن إضافة السماد البوتاسي أدى ايضا إلى حدوث زيادة معنوية في متوسط عدد تفر عات النيات النبات بزيادة

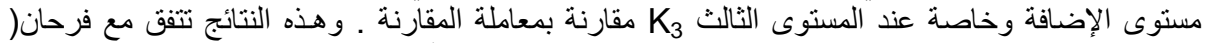
2012 ) إذ وجدا إن الإضافات الجيدة من البوتاسيوم لنباتات الفول أدت إلى زئل زيادة معنوية في عدد الفئة الفروع

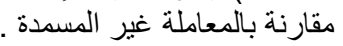

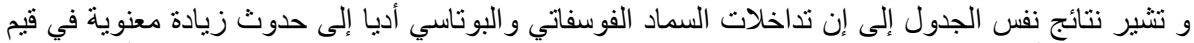

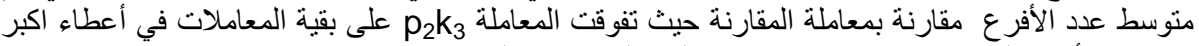

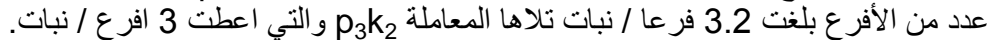

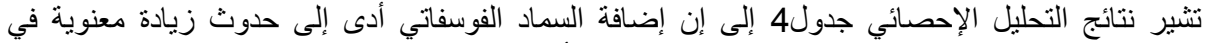

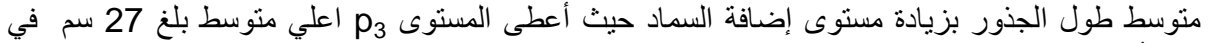
حين أعطت معاملة المقارنة 24.08 سم وقد يعزى السئ السبب في ذللك إلى إن الفوسفور يؤدى إلى زئى زيادة استطالة

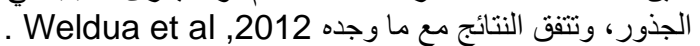

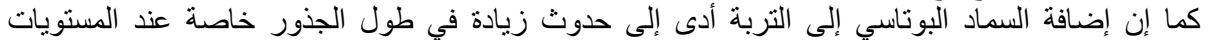

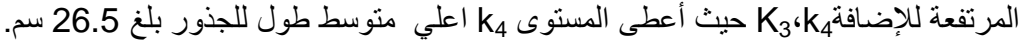


جذول 4 : تأثير إضافة السماد الفوسفاتي والبوتاسي وتداخلاتهما على طول الجذور سم P1

\begin{tabular}{|c|c|c|c|r|}
\hline Mean K & P3 & P2 & P1 & Treatment \\
\hline 24.87 & 26.6 & 26 & 22 & K1 \\
\hline 24.37 & 25.5 & 24.3 & 23.3 & K2 \\
\hline 26 & 26.9 & 26.2 & 25 & K3 \\
\hline 26.5 & 29 & 24.5 & 26 & K4 \\
\hline 0.90 & 27 & 1.59 & Mean P \\
\hline & 25.25 & 24.08 & LSD \\
\cline { 2 - 5 }
\end{tabular}

كما إن تداخلات السماد الفوسفاتي مع السماد البوتاسي أدت إلى زيادة منوسط أطوال جذور

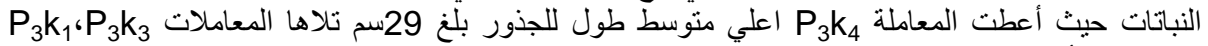

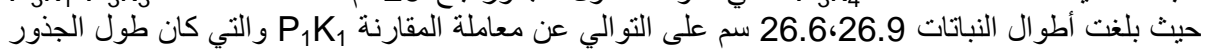

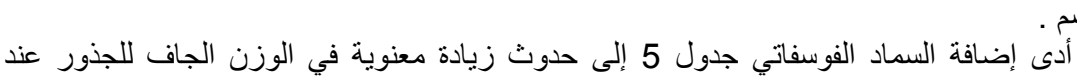

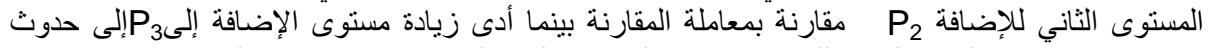

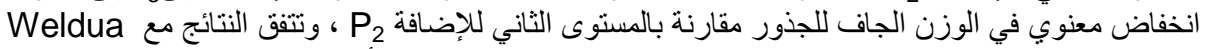

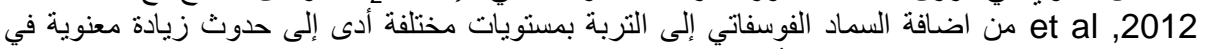

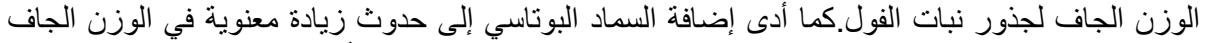

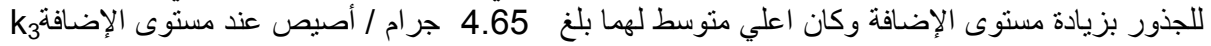

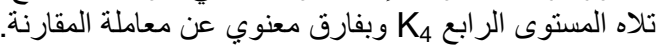

جدول 5: تأثير إضافة السماد الفوسفاتي والبوتاسي وتداخلاتهما على متوسط الوزن الجاف لجذور نباتات

\begin{tabular}{|c|c|c|c|c|}
\hline & & \multicolumn{3}{|c|}{ الفول ـ( جرام/أصيص) } \\
\hline Mean K & P3 & P2 & P1 & Treatment \\
\hline 4.20 & 3.68 & 4.75 & 4.18 & K1 \\
\hline 4.34 & 4.16 & 4.62 & 4.24 & $\mathrm{~K} 2$ \\
\hline 4.65 & 4.88 & 4.82 & 4.26 & $\mathrm{~K} 3$ \\
\hline 4.51 & 4.82 & 4.39 & 4.33 & $\mathrm{~K} 4$ \\
\hline 0.05 & \multicolumn{3}{|c|}{0.09} & LSD \\
\hline & 4.39 & 4.65 & 4.25 & Mean P \\
\hline & \multicolumn{3}{|c|}{0.07} & LSD \\
\hline
\end{tabular}

كما تشير نتائج نفس الجدول إلى ان تداخلات الأسمدة أدت إلى حدوث زيادة معنوية في الوزن

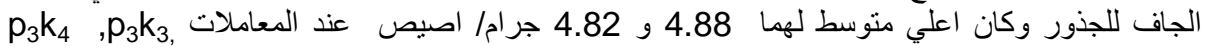

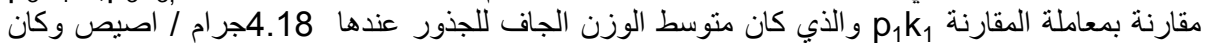

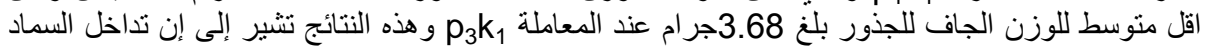

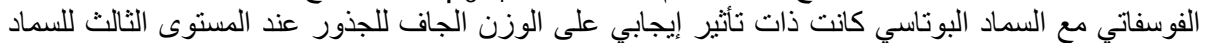

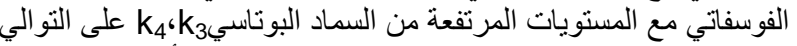

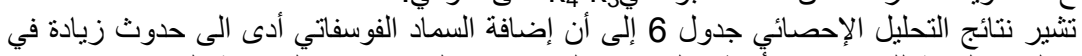

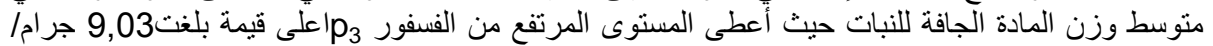

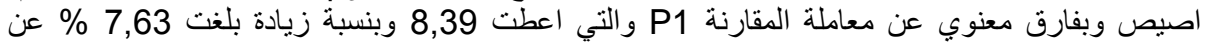
معاملة المقارنة وهذا يتو افق مع ما وجدة (Mohamed,2005)و .et al ,2012

كما أدى إضافة السماد البوتاسي إلى حدوث زيادة في منوسط وزن المادي المادة الجافة للنبات بزيادة

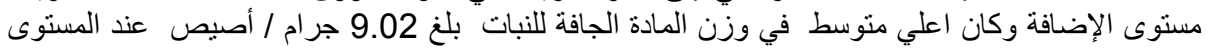




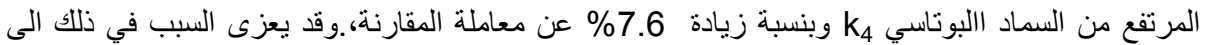

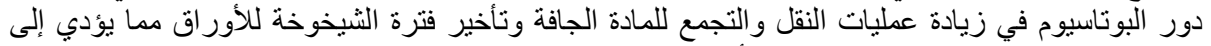

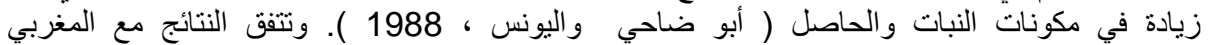
و الدساوي(2012) فلي الي الذرة الثامية و(Zakaria and EL zemrany 2012) علي القمح و فرحان

$$
\text { (2012) علي الفول. (2012) }
$$

جدول 6: تأثير إضافة السماد الفوسفاتي والبوتاسي وتداخلاتهما على متوسط وزن المادة الجافة لنباتات

\begin{tabular}{|c|c|c|c|c|}
\hline & & \multicolumn{3}{|c|}{ الفول ( جراج/ أصيص). } \\
\hline Mean K & P3 & P2 & P1 & Treatment \\
\hline 8.38 & 8.51 & 8.35 & 8.28 & K1 \\
\hline 8.63 & 9.14 & 8.43 & 8.31 & K2 \\
\hline 8.66 & 9.17 & 8.48 & 8.34 & K3 \\
\hline 9.02 & 9.31 & 9.14 & 8.62 & K4 \\
\hline 0.632 & \multicolumn{3}{|c|}{0.835} & LSD \\
\hline & 9.03 & 8.60 & 8.39 & Mean $\mathrm{P}$ \\
\hline & \multicolumn{3}{|c|}{0.634} & LSD \\
\hline
\end{tabular}

كما تثنير نتائج نفس الجدول إلى إن تداخل السماد الفوسفاتي والبوتاسي أديا إلى حدوث زيادة الجادة

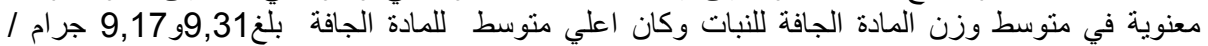

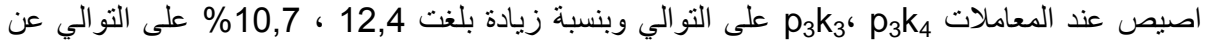
معاملة المقارنة ولم تكن الفروق معنوية فيما بينها.

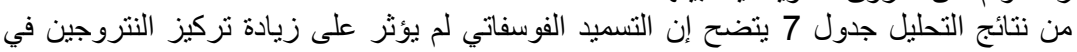

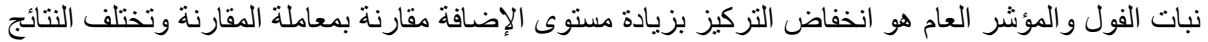
مع ما وجدة (Mohamed,2005).وربما يعزي ذلك الي زيادة كمية المادة الجافة نتيجة لإضافة مستويات التسميد الفوسفاتي مجدة

جدول 7 : تأثير إضافة السماد الفوسفاتي والبوتاسي وتداخلاتهما على متوسط تركيز النيتروجين في النبات

\begin{tabular}{|c|c|c|c|r|}
\hline Mean K & P3 & P2 & P1 & Treatment \\
\hline 2,55 & 2,65 & 2,44 & 2,56 & K1 \\
\hline 2,67 & 2,69 & 2,68 & 63,2 & K2 \\
\hline 2,73 & 2,77 & 2,71 & 2,71 & K3 \\
\hline 2,59 & 2,48 & 2,46 & 2,84 & K4 \\
\hline 0.06 & \multicolumn{3}{|c|}{0.12} & LSD \\
\hline & 2.65 & 2.57 & 2.68 & Mean P \\
\hline \multicolumn{4}{|c|}{0.10} & LSD \\
\hline
\end{tabular}

بينما أدى إضافة التسميد البوتاسي إلى حدوث زيادة معنوية في تركيز النيتزوجين بزيادة مستويات

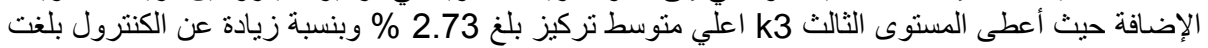

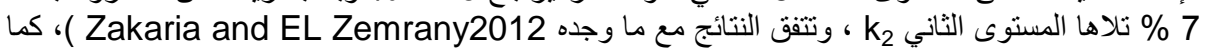

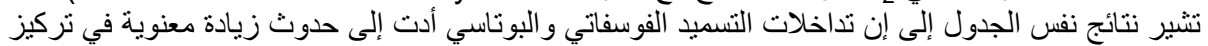

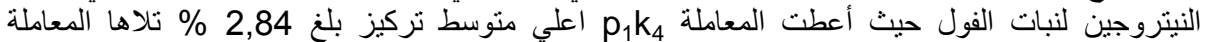

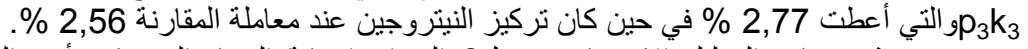

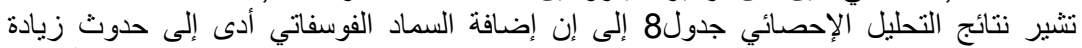

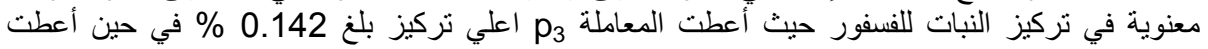

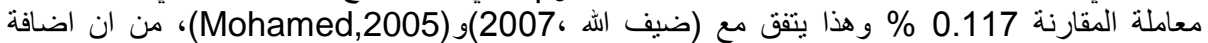
السماد الفوسفاتي ادى الى حدوث زيادة معنوية في محنوى النبات من الفوسفور ، 
جاول8 : تأثير إضافة السماد الفوسفاتي والبوتاسي وتداخلاتهما على تركيز الفسفور في النبات \%

\begin{tabular}{|c|c|c|c|r|}
\hline Mean K & P3 & P2 & P1 & Treatment \\
\hline 0.119 & 0.126 & 0.125 & 0.106 & K1 \\
\hline 0.135 & 0.164 & 0.126 & 0.115 & K2 \\
\hline 0.137 & 0.163 & 0.124 & 0.123 & K3 \\
\hline 0.122 & 0.116 & 0.124 & 0.125 & K4 \\
\hline 0.005 & 0.142 & 0.125 & 0.117 & Mean P \\
\hline & 0.009 & LSD \\
\cline { 2 - 5 }
\end{tabular}

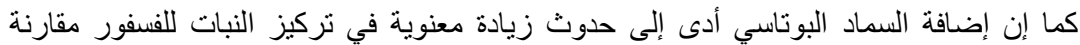
بمعاملة المقارنة حيث أعطى المستوى الثالث k

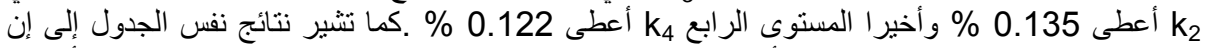

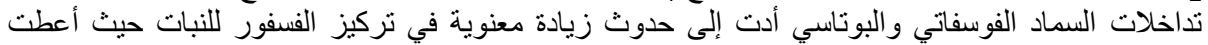

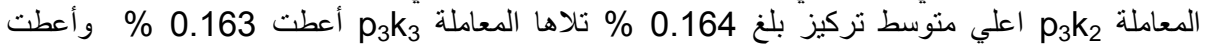

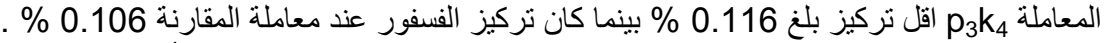

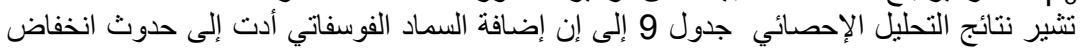

في تركيز البوتاسيوم في نبات الفول . لفيل

جدول 9: تأثير إضافة السماد الفوسفاتي والبوتاسي وتداخلاتهما على تركيز البوتاسيوم في النبات P3

\begin{tabular}{|c|c|c|c|r|}
\hline Mean K & P3 & P2 & P1 & Treatment \\
\hline 1.67 & 1.56 & 1.62 & 1.82 & K1 \\
\hline 1.44 & 1.35 & 1.47 & 1.51 & K2 \\
\hline 1.58 & 1.57 & 1.55 & 1.62 & K3 \\
\hline 1.85 & 2.01 & 1.62 & 1.92 & K4 \\
\hline 0.021 & 1.62 & 0.036 & LSD \\
\hline & \multicolumn{4}{|c|}{0.025} \\
\cline { 2 - 5 } & 1.56 & 1.72 & LSD \\
\hline
\end{tabular}

كما إن إضافة السماد البوتاسي أدت إلى حدوث زيادة معنوية في تركيز البوتاسيوم في النبات حيث

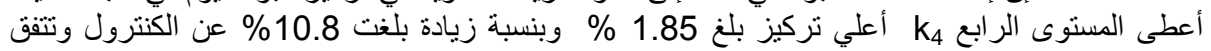

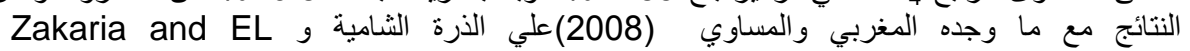

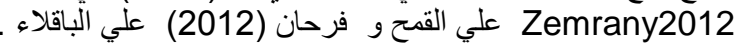

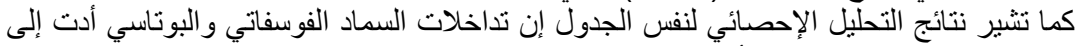

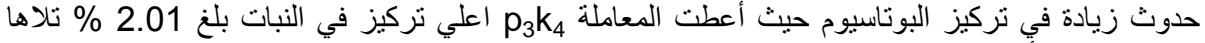

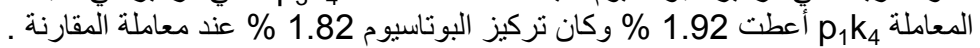




$$
\text { المراجع }
$$

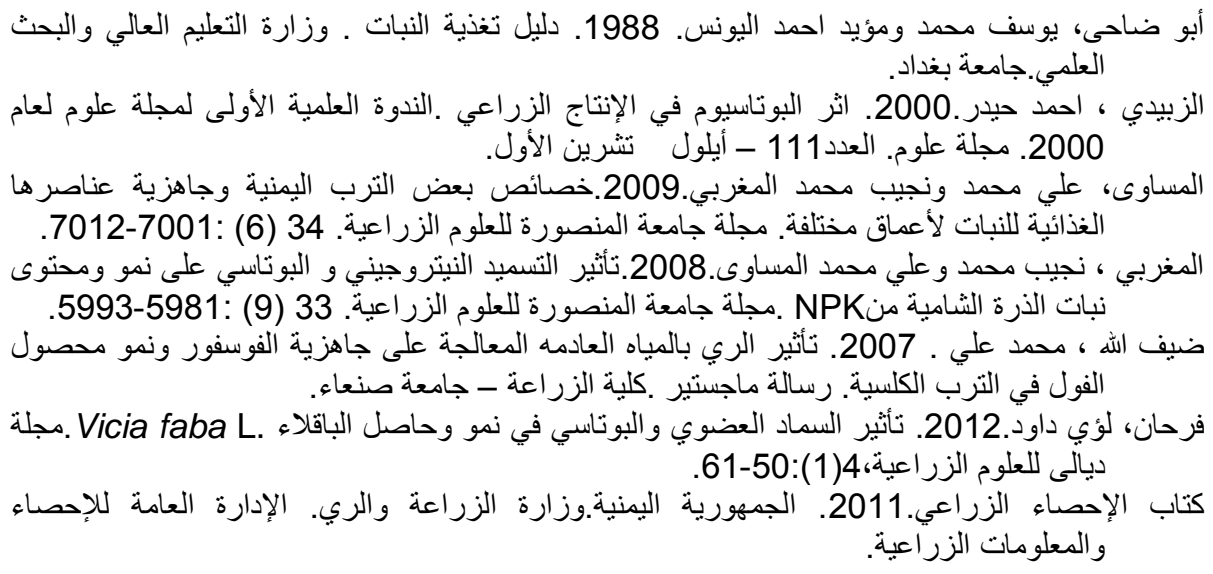

Agegnehu.G; Tsig, A.( 2006).The role of phosphorus fertilization on growth and yield of faba bean on acidic Nitisol of central highland of Ethiopia. Ethiopian Journal of Science. 29( 2):177-182.

Chafi, M.H. and A. Bensoltane (2009). Vicia faba (L), A source of organic and biological manure for the Algerian Arid Regions.

World Journal Agriculture Science 5(6):698-706.

Hashemabadi, D.(2013): Phosphorus fertilizers effect on the yield and yield components of faba bean (Vicia faba L). Annals of Biological Research; 2013. 4( 2): 181-184.

Jalali M.and M.Zarabi (2006). Kinetics of nonexchangeable-potassium release and plant response in some calcareous soils. J.Pant Nutr.and Soil Sci.169(2):196-204.

Kandil,Hala(2007). Effect of cobalt fertilizers on growth ,yield and nutrient status of faba bean(Vicia faba L.) plant. Journal of Applied Science Research 3(9):867-872.

Mohamed,Gamal.A(.2005).Yield and yield components of faba bean (Vicia faba,L. ) as affected by phosphorus and nitrogen fertilztion levels. Assiut . J. .Agric.Sci.36 (6):111-122.

Page,A.L.;R.h.Mill and D.R.Keeney(1982).Method of Soil Analysis Part2.Madison,Wisconsin.U.S.A.

Weldua.Y ; Haileb. M; Habtegebrielb. K.(2012). Effect of zinc and phosphorus fertilizers application on yield and yield components of faba bean (Vicia faba L.) grown in calcaric cambisol of semi-arid northern Ethiopia. Journal of Soil Science and Environmental Management. 3( 12): 320326.

Zakaria ,M.Sahar.,and H.M. EL Zemrany (2012). Eficiency of potassium fertilization for wheat grown on saline aoil as affected by biofertilization and compost Application. Minufiya J.Agric.Res.37(2):409-421. 


\title{
THE EFFECT OF DIFFERENT LEVELS OF POTASSIUM AND PHOSPHATE FERTILIZER ON GROWTH AND NITROGEN ,PHOSPHORUS AND POTASSIUM CONTENTS OF BEAN PLANT (VICIA FABA L ) \\ Najeeb Mohamed Hussein ALmagrebi ${ }^{2}$ \\ Soil and Water Dept. ,Fac. of Agric. ,Sana Univ. , Yemen
}

\begin{abstract}
A pot experiment was carried out in the greenhouse of Soil and Water Dept. Faculty of Agriculture, University of Sana'a during 2008 season on a sedimentary salty clay soil, to study the effect of different levels of potassium and phosphate fertilizer in the growth and NPK contents of bean plants. The experiment was designed using a split plot design with three replications where phosphate levels were the main plots and potassium fertilization levels were the sub plots . Three levels of phosphate fertilizer, (superphosphate triple $21 \% \mathrm{P}, 0.60,120 \mathrm{~kg} \mathrm{P} /$ ha.).were examined and have been given the following codes (P3, P2, P1), respectively. Four levels of potassium fertilizer (potassium sulfate, $42 \% \mathrm{~K} ; 0,50,100$, $150 \mathrm{~kg} \mathrm{~K} / \mathrm{ha}$. ) were also used and have been given the following codes (K4, K3, K2 $, \mathrm{K} 1)$, respectively. Nitrogen fertilizer in the form of urea was added for all transactions at the rate of $60 \mathrm{~kg} \mathrm{~N} /$ ha after germination. The third level (P3) of phosphate fertilizer (120 kg / ha) was significantly increased plant height and number of branches / plant and root length and weight of the dry matter, while the the second level (60 kg P / ha) had the superiority to increase dry weight of roots. Also add phosphate fertilizer caused a significant increase in the concentration of $P$ in plant tissues while insignificantly influence on the nitrogen content of the plant was detected. Meanwhile, potassium content of the plant was reduced due to the addition of $P$ levels. Add potassium fertilizer led to a significant increase in the aforementioned diameters and the best significant effect of potassium fertilizer was recorded at the third level (100 kg K/ ha) and fourth (150 kg K/ ha). Resulted data showed that the interaction between potassium and phosphate treatments gave significant increases in the studied parameters, the treatment P3K4 highest produced the highest values of plant height, dry matter, root length and the concentration of potassium in the plant; while the treatment P3K3 resulted in a significant increase in dry weight of roots and content of nitrogen. P3K2 treatment led to significant increase in the phosphorus content of the plant.

Keywords:Yamen soil, fertilizer, potassium, bean, phosphorus, content of plant, plant growth, nitrogen
\end{abstract}

Associate Professor, Department of Soil and water, Faculty of Agriculture, University of ${ }^{2}$ Sana'a, Yemen 